\title{
Continuous wound infusion with chloroprocaine in a pig model of surgical lesion: drug absorption and effects on inflammatory response
}

This article was published in the following Dove Press journal:

Journal of Pain Research

31 October 2017

Number of times this article has been viewed

\author{
Massimo Allegri, ${ }^{1,2}$ Dario \\ Bugada, ${ }^{1-3}$ Manuela De \\ Gregori, ${ }^{2,4}$ Maria A Avanzini, ${ }^{5}$ \\ Annalisa De Silvestri, ${ }^{6}$ Anna \\ Petroni, ${ }^{7}$ Angelo Sala, ${ }^{7,8}$ \\ Claudia Filisetti, ${ }^{9-11}$ Antonia \\ Icaro Cornaglia, ${ }^{12}$ Lorenzo \\ Cobianchi ${ }^{13,14}$
}

'Department of Medicine and Surgery, University of Parma, Parma ${ }^{2}$ SIMPAR

Group (Study in Multidisciplinary

PAin Research), ${ }^{3}$ Department of

Anaesthesia and ICU, ASST Papa

Giovanni XXIII, Bergamo, ${ }^{4}$ Pain

Therapy Service, Fondazione IRCCS

Policlinico San Matteo, ${ }^{5}$ Laboratory

of Transplant Immunology/Cell

Factory, IRCCS Foundation Policlinico

San Matteo, ${ }^{6} \mathrm{Clinical}$ epidemiology

and Biometrics Unit, Fondazione

IRCCS Policlinico San Matteo, Pavia,

${ }^{7}$ Department of Pharmacological and

Biomolecular Sciences, University

of Milan, Milan, ${ }^{8}$ I.B.I.M., C.N.R.,

Palermo, ${ }^{9} \mathrm{PhD}$ School, University

of Pavia, ${ }^{10}$ Department of Pediatric

Surgery, Fondazione IRCCS Policlinico

San Matteo, Pavia, "Department of

Pediatric Surgery, "V. Buzzi" Children's

Hospital, Milan, ${ }^{2}$ Department of

Public Health, Experimental and

Forensic Medicine, University of Pavia,

${ }^{13}$ Department of Surgical, Clinical,

Paediatric and Diagnostic Science,

University of Pavia, ${ }^{14}$ General Surgery

I, IRCCS Fondazione Policlinico San

Matteo, Pavia, Italy

Correspondence: Lorenzo Cobianchi Department of Surgical, Clinical, Pediatric and Diagnostic Science, University of Pavia, and General Surgery I, IRCCS Fondazione Policlinico San Matteo, P.LE Golgi 19, 27100 Pavia,

Email l.cobianchi@smatteo.pv.it
Abstract: Continuous wound infusion (CWI) may protect from inflammation, hyperalgesia and persistent pain. Current local anesthetics display suboptimal pharmacokinetic profile during CWI; chloroprocaine (CP) has ideal characteristics, but has never been tested for CWI. We performed an animal study to investigate the pharmacokinetic profile and anti-inflammatory effect of CP during CWI. A total of 14 piglets received an infusion catheter after pararectal laparotomy and were randomly allocated to one of three groups: $5 \mathrm{~mL} / \mathrm{h}$ infusion of saline (group A), CP $1.5 \%$ (group B) and CP $0.5 \%$ (group C). Blood sampling was performed to assess absorption and systemic inflammation at $0,3,6,12,24,48,72,96,102$ and 108 hours. The wound and contralateral healthy abdominal wall were sampled for histological analyses. Absorption of CP from the site of infusion, evaluated as the plasmatic concentrations of $\mathrm{CP}$ and its metabolite, 4-amino-2-chlorobenzoic acid (CABA), showed a peak during the first 6 hours, but both CP and its metabolite rapidly disappeared after stopping $\mathrm{CP}$ infusion. Local inflammation was reduced in groups B and C (CP-treated $p<0.001)$, in a CP dose-dependent fashion. While CP inhibited in a dose-dependent manner pig mononuclear cells (MNCs) in vitro proliferation to a polyclonal activator, no effect on systemic cytokines' concentrations or on ex vivo monocytes' responsiveness was observed, suggesting the lack of systemic effects, in line with the very short half-life of $\mathrm{CP}$ in plasma. CP showed a very good profile for use in CWI, with dose-dependent local anti-inflammatory effects, limited absorption and rapid clearance from the bloodstream upon discontinuation. No cytotoxicity or side effects were observed. CP, therefore, may represent an optimal choice for clinical CWI, adaptable to each patient's need, and protective on wound inflammatory response (and hyperalgesia) after surgery.

Keywords: continuous wound infusion, pig model, chloroprocaine, pharmacokinetics, inflammation, postoperative pain

\section{Introduction}

Postoperative analgesia still remains a challenge, ${ }^{1,2}$ and the importance of targeted treatments has often been stressed. ${ }^{3,4}$ Pain involves different mechanisms (peripheral nociception, central modulation and primary and secondary hyperalgesia), with nociceptor's activation being the trigger for all. Inflammation is the major background factor acting both peripherally and centrally on neural structures, leading to hyperalgesia and probably acting as a main factor toward the development of persistent pain after surgery. ${ }^{5,6}$

Since the choice of the analgesic strategy should be targeted on pain pathophysiology, ${ }^{7}$ a rational approach to postoperative analgesia is to block the origin of pain cascade (namely peripheral nociception) and to prevent all pain-related events. Thus, 
further to subjective pain relief, an effective, prolonged $^{8}$ nociceptive block should reduce inflammatory response ${ }^{9}$ and central sensitization. ${ }^{10,11}$

Continuous wound infusion (CWI) with local anesthetics (LAs) has been documented as an effective technique for postoperative pain relief in several studies ${ }^{12}$ and comparable to epidural analgesia. ${ }^{13}$ LAs display local/systemic anti-inflammatory properties, even at low concentrations. ${ }^{14}$ LAs reduce polymorphonuclear granulocytes' (PMNs) adhesion in the surgical site and block leukotriene B4, a powerful chemotactic mediator. Lidocaine and ropivacaine reduce TNF $\alpha$-induced upregulation of integrins (CD11bCD18) essential for endothelial adhesion and diapedesis into the injured tissue; lidocaine and tetracaine cause loss of PMNs' round shape necessary for adhesion. The global effect is the reduction of PMNs' accumulation in the surgical wound, together with the release of local inflammatory chemokines. ${ }^{14}$

Currently, drugs used for CWI are long-lasting LAs, such as ropivacaine, bupivacaine, and levobupivacaine. Such drugs are considered safe at clinical doses, and the kinetic profile and blood levels have already been investigated during epidural infusions. ${ }^{15}$

However, both from a clinical and a pharmacokinetic perspective, an ultra short-acting LA may be more appropriate for CWI, as it could be better modulated according to specific patients' needs with "on/off" profile.

Chloroprocaine (CP) has fast onset (6-10 minutes) and short action (around 90 minutes), ${ }^{16}$ with a cholinesterasebased plasmatic metabolism. Early cases of toxicity after spinal anesthesia ${ }^{17}$ were documented, but were related to the sodium bisulfite antioxidant and to the solution's low $\mathrm{pH} .{ }^{18}$ Nowadays, antioxidant-free, safer preparations are available, and interest has grown again because of CP's ideal profile for CWI.

There is no study, to our knowledge, investigating CP during intrawound continuous infusion in an animal model; moreover, there are few data elucidating the effect on local and systemic inflammation of a continuous subfascial wound infusion of LAs and in particular for CP.

As recently underlined by Gigliuto et al, ${ }^{19}$ a large animal model could be more appropriate than others to produce reliable translational results. We therefore evaluated the absorption associated with a continuous $\mathrm{CP}$ intrawound (subfascial) infusion in pig. We also investigated if $\mathrm{CP}$ at two different dosages may influence local and systemic inflammatory responses after surgical lesion both in vivo and ex vivo.

\section{Materials and methods}

All animal procedures were performed according to the protocol approved by Institutional Animal Care and Use Committee (IACUC) - University of Pavia. All animal care and experimental procedures followed local, national and European Union guidelines for the animals' use (Directive 2010/63/EU - revising Directive 86/609/EEC on the protection of animals used for scientific purposes).

\section{Animals and surgical procedures}

Piglets $(20-30 \mathrm{~kg})$ underwent pararectal laparotomy, extended for $10 \mathrm{~cm}$. The parietal peritoneal membrane was then closed with a continuous absorbable synthetic suture. An intrawound, multiholed catheter (PAINFusor ${ }^{\circledR}$ - Plan1-Health, Udine, Italy) was placed between peritoneum and deep muscular fascia along the length of the wound and tunneled subcutaneously. Muscle layers and skin were finally closed with a continuous absorbable synthetic suture.

Surgical procedure was performed under general balanced anesthesia, including mechanical ventilation via orotracheal intubation and mioresolution. ${ }^{20,21}$ A central venous catheter was placed in the right external jugular vein with open surgical technique and was used for drug infusion and for blood sampling. At the end of surgical procedures, piglets were set in the metabolic cage and were supplied with water and standard bran. Intravenous antibiotic (cefazolin $1 \mathrm{~g}$ ) and omeprazole $40 \mathrm{mg}$ were administered during follow-up to prevent wound infections and stress-induced gastric ulcerations. Analgesia was performed in all animals by intramuscular injection of tramadol $50 \mathrm{mg}$ during the perioperative period, with no additional analgesics, according to our animal protocols for surgical trials.

Animals were randomly allocated to 3 groups with a $5 \mathrm{~mL} / \mathrm{h}$ continuous infusion through elastomeric pump for 72 hours: saline (group A), CP 1.5\% (group B) or CP 0.5\% (group C). The randomization was based on a computer-generated list.

\section{Sample collection}

During the follow-up period, peripheral blood was collected for the determination of $\mathrm{CP}$ and its metabolite, as well as of the effects on systemic inflammation, at $0,3,6,12,24,48$, $72,96,102$ and 108 hours from incision. Plasma was obtained by centrifugation at $3000 \mathrm{rpm}$ for 15 minutes and stored at $-20^{\circ} \mathrm{C}$ until analysis. Mononuclear cells (MNCs), for in vitro and ex vivo experiments, were obtained by gradient density on cell separation media (Cedarlane, Burlington, USA) at $2500 \mathrm{rpm}$ for 20 minutes. The catheter was removed after 
96 hours of infusion. Piglets were finally sacrificed, according to international guidelines.

\section{Determination of CP and its metabolite in plasma}

$\mathrm{CP}$ and its metabolite 4-amino-2-chlorobenzoic acid (CABA) were analyzed by liquid chromatography/tandem mass spectrometry using a high performance liquid chromatography (HPLC) Agilent (model 1200) equipped with an autosampler (PAL HTS-xt; PAL System, CTC Analytics, Zwingen, Switzerland) directly interfaced with a triple-quadruple API 4000 (AB Sciex, Framingham, MA, USA), equipped with an electrospray ionization (ESI) source. ${ }^{22,23} \mathrm{CP}$ was provided by Sintetica SrL, (Mendrisio, Switzerland) CABA by Sigma-Aldrich Co. (St Louis, MO, USA) and deuterated PABA (4-aminobenzoic acid), used as internal standard (IS) for chloroprocaine and CABA quantitation, by Santa Cruz Biotechnology Inc., Dallas, TX, USA. Methanol and formic acid were from Sigma-Aldrich, whereas HPLC grade water was obtained using a Milli Q water purification system. Chromatographic separation was carried out using a Synergi 4 micro-Hydro-RP $80 \mathrm{~A}, 150 \times 2 \mathrm{~mm} \times 4 \mu \mathrm{m}$ column (Phenomenex, Torrance, CA, USA) at a flow rate of $200 \mu \mathrm{L} /$ min. A total of $10 \mu \mathrm{L}$ of the sample was injected in a linear gradient system from $85 \%$ of solvent $\mathrm{A}(0.1 \%$ formic acid in water) to $90 \%$ of solvent $\mathrm{B}(0.1 \%$ formic acid in methanol) over 3 minutes. Final conditions were held for 3 minutes before equilibration back at starting conditions.

The triple-quadruple was operated in positive ions mode for multiple reaction monitoring (MRM). The following characteristic fragmentations were used for identification and quantitative analyses: CP from m/z 272 to 198.6 or 153.9 , CABA from $\mathrm{m} / \mathrm{z} 172.2$ to 128 or 153.9 , and $\mathrm{d}_{4}$-PABA from $\mathrm{m} / \mathrm{z} 142.1$ to 98.8 or 123.9 . The most abundant transition was used for quantitative analysis, while the second transition was used as qualifier for additional identification purposes. A total of $100 \mu \mathrm{L}$ of plasma samples were added with $20 \mu \mathrm{L}(1 \mathrm{ng} / \mu \mathrm{L})$ IS and $400 \mu \mathrm{L}$ of methanol; samples were then put at $-20^{\circ} \mathrm{C}$ overnight. After centrifugation at 13,000 rpm for 10 minutes, $450 \mu \mathrm{L}$ of the solution were recovered and evaporated using a rotary evaporator. Finally, the samples were resuspended in $100 \mu \mathrm{L}$ of water/methanol (1:1) and $10 \mu \mathrm{L}$ was used for liquid chromatography/tandem mass spectrometry analysis. The calibration curves were prepared in drug-free pig plasma, from working solutions of CP and CABA of 1, 3, $10,30,100$ and $300 \mathrm{ng} / \mathrm{mL}$ and processed as the biological samples. Calibration curves were obtained plotting the ratio of the chromatographic area of specific transitions for the compounds of interest over that of specific transition of the internal standard, and the correlation coefficient was always better than 0.99 . The limit of quantification (LOQ) was $1 \mathrm{ng} /$ $\mathrm{mL}$ of plasma for both compounds.

Data were acquired and processed using Analyst 1.6.1. Concentrations are presented as mean and standard error of the mean of $n$ determinations.

\section{Histopathological analysis}

For histological examination, 3 samples of the full-thickness wound and 1 sample of the contralateral healthy abdominal wall were collected from each animal. Each sample was $0.5 \mathrm{~cm}$ thick. The samples were fixed in $4 \%$ neutral buffered formalin for 48 hours, dehydrated with gradient alcohol series, cleared in xylene and eventually embedded in paraffin. Serial sections $(8 \mu \mathrm{m})$ were obtained using a Leitz (Wetzlar, Germany) microtome, stained with hematoxylin and eosin (H\&E) and examined with a Zeiss (Oberkochen, Germany) Axiophot microscope.

We evaluated the presence of inflammatory cells: neutrophils, eosinophils, lymphocytes, macrophages and mast cells, in pericatheter blank tissues. A total of 15 sections were evaluated for statistical analysis ( 4 sections per group - corresponding to a different animal of each experimental group plus 1 contralateral healthy sample per group). For each section, at least 8 randomly determined areas of connective tissue were examined at $20 x$ magnification and 100 cells per area were counted. The rate of inflammatory cells was assessed using a semiquantitative scale, with scores ranging from 0 to 4 by 3 independent observers (Table 1). Histological scores are reported as median and 25-75 percentile (interquartile range, IQR).

\section{Systemic inflammation analysis}

Systemic inflammation was evaluated by quantifying pig interleukin-6 (pIL-6), pIL-10, pIL-8, pIL-1 $\beta$ and pig interferon (pIFN)- $\gamma$ levels in plasma samples at each time point, after incision in all animals, by commercially available quantitative enzyme-linked immunosorbent assay (ELISA) (Quantikine; R\&D Systems, Inc., Minneapolis, MN, USA). Detection limits were as follows: $18.8 \mathrm{pg} / \mathrm{mL}$ for pIL-6, $31.3 \mathrm{pg} / \mathrm{mL}$ for $\mathrm{pIL}-10,62.5 \mathrm{pg} / \mathrm{mL}$ for $\mathrm{pIL}-8,39.1 \mathrm{pg} / \mathrm{mL}$ for $\mathrm{pIL}-1 \beta$ and $39 \mathrm{pg} / \mathrm{mL}$ for $\mathrm{pIFN}-\gamma$.

Table I Semiquantitative score used for the assessment of the percentage of the inflammatory infiltrate

\begin{tabular}{ll}
\hline Score & Percentage of inflammatory infiltrate \\
\hline 0 & $0-10$ \\
1 & $10-25$ \\
2 & $25-50$ \\
3 & $50-75$ \\
4 & $>75$ \\
\hline
\end{tabular}




\section{In vitro and ex vivo cell proliferation suppression}

In order to evaluate in vitro cell proliferation suppression, $\mathrm{CP}$ and CABA were added at different concentrations (from 1.25 to $0.02 \mathrm{mg} / \mathrm{mL}$ ) to resting and phytohemagglutinin (PHA)activated MNCs obtained before treatment. In detail, $10^{5}$ $\mathrm{MNCs} /$ well were plated in triplicate in flat-bottom microwells (Corning Costar, Euroclone, Milan, Italy), in RPMI 1640 medium (ThermoFisher Scientific, Waltham, MA, USA), $10 \%$ fetal calf serum (Euroclone, Celbio), without (resting) or with PHA (activated) (4 g/mL; Boehringer Ingelheim, Mannheim, Germany). After a 3-day incubation at $37^{\circ} \mathrm{C}$ in a humidified 5\% $\mathrm{CO}_{2}$ atmosphere, ${ }^{3} \mathrm{H}$-thymidine $\left({ }^{3} \mathrm{HTdR} 0.5\right.$ $\mathrm{mCi}$ /well; Amersham, Buckinghamshire, UK) incorporation was measured during the last 21 hours by standard procedure. Results were expressed as stimulation index (SI = cpm stimulated/cpm unstimulated) and reported as residual proliferation.

Moreover, in order to define if $\mathrm{CP}$ in vivo treatment induced a change in cell response capability we evaluated ex vivo, with the same methodology, the effects of CP treatment on the response to PHA of MNCs obtained at different time points (basal, 96 and 108 hours) of pharmacological treatment in groups A (saline), B (CP 1.5\%) and C (CP 0.5\%).

\section{Cell viability}

To test if $\mathrm{CP}$ and CABA could affect MNCs viability, after in vitro culture, cells were evaluated by Trypan blue (Sigma, Milan, Italy) exclusion test. Percentage of cell viability is calculated as (number of viable cells/total number of cells [viable + nonviable $] \times 100)$.

\section{Statistical analysis}

Cytokine concentrations were compared between groups with analysis of variance for repeated measure. An ordinal logistic regression model was fitted using histological score as dependent variable and group as independent variable. To compare all groups, post hoc estimation (between doses or between groups $\mathrm{B}$ and $\mathrm{C}$ and group A) and Wald tests were also performed. A $p$-value $<0.05$ was considered statistically significant.

Quantitative variables are described as mean and SD. Comparisons between groups (A and B, C) and time were performed by means of analysis of variance for repeated measures. The same method was applied to analyze SI at different $\mathrm{CP}$ and $\mathrm{CABA}$ concentrations. Robust standard errors were considered to take into account the clustered nature of the data. A $p$-value $<0.05$ was considered significant. Stata v14.1 (StataCorp LP, College Station, TX, USA) was used for all the analyses.
We did not perform a power analysis since previous literature does not provide data to support any power calculation. The present study is to be considered as a pilot study.

\section{Results}

Four animals (25-30 kg, average weight $29.6 \mathrm{~kg}$ ) were randomly allocated to each group; in group A 2 additional animals were studied as 2 animals were excluded as they developed fever and wound infections. All the other animals in groups B and C completed the follow-up period uneventfully, and they were all treated without any deviation from the protocol.

We did not register any adverse effect or allergic reaction in all the animals used for the study.

\section{Concentrations of $\mathrm{CP}$ and its metabolites in plasma}

Concentrations of CP were mostly below the quantitation limit, with only 6 blood samples showing the presence of very low concentrations of the intact molecule (1-2.3 ng/ $\mathrm{mL}$ in animals perfused with $\mathrm{CP} 0.5 \%$, and $1-20.8 \mathrm{ng} / \mathrm{mL}$ in animals perfused with $\mathrm{CP} 1.5 \%$ ). CABA concentrations showed a sharp increase immediately after the infusion was started, peaking between 3 and 6 hours from the beginning of the infusion, and returning to basal level (zero) as soon as the continuous infusion was stopped (Figure 1). The peak in CABA concentrations appeared to be proportional to the concentration of CP infused (Figure 1), averaging 177 \pm 141 and $543 \pm 169 \mathrm{ng} / \mathrm{mL}$ in animals perfused with $\mathrm{CP} 0.5 \%$ and $1.5 \%$, respectively.

\section{Histopathological analysis}

The presence of inflammatory cells was significantly higher in group A than in groups B and C ( $p<0.001$, in both cases). These results are well summarized by semiquantitative scores (Table 2) that show a dose-dependent reduction of the inflammatory infiltrate within the wound, which is nearly halved with higher CP doses.

This reduction is even more evident in wound sections, where refined analyses on tissue damage and reaction are possible: pigs in group A (saline) have high amounts of leukocytes and lymphocytes infiltrating wound layers up to connective tissue, and cells surrounding the catheter's blank display signs of necrosis (Figure 2). Signs of cell damage are less evident in $\mathrm{CP}$-infused pigs, and inflammatory infiltrates are less pronounced and different in nature (less leukocytes); inflammation reaches the connective tissue to a lesser extent, and signs suggestive of tissue regeneration are 


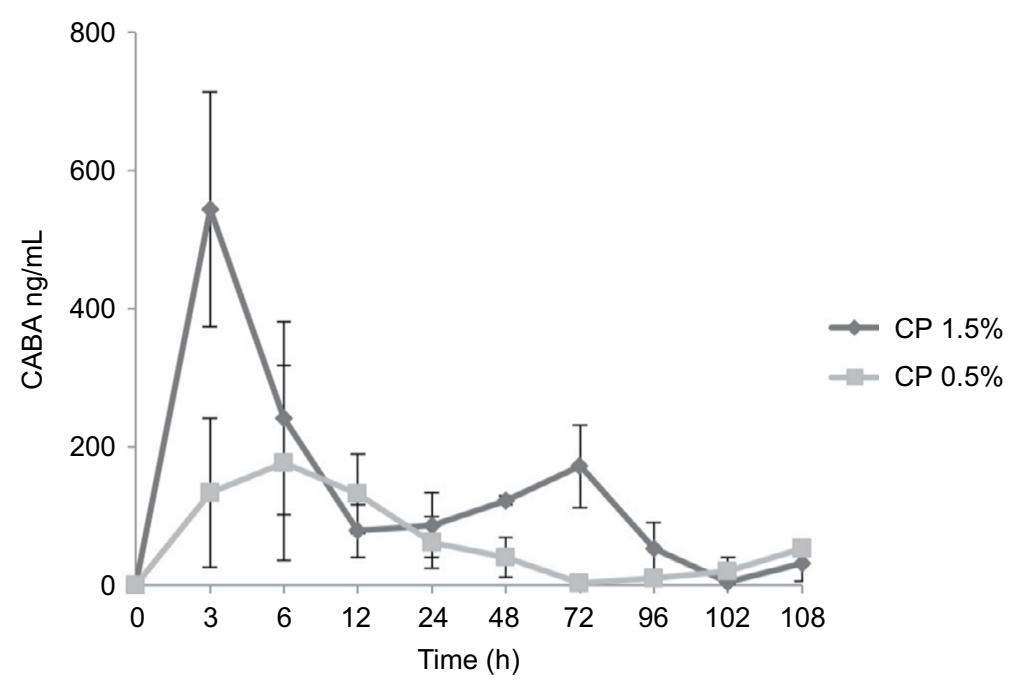

Figure I CABA plasma concentrations after continuous infusion with $0.5 \%$ (closed squares) and I.5\% (closed diamond) of chloroprocaine.

Note: CABA was measured by liquid chromatography/tandem mass spectrometry as described in the "Materials and methods" section. Data are expressed as mean and $\operatorname{SEM}(n=4)$.

Abbreviations: CABA, 4-amino-2-chlorobenzoic acid; CP, chloroprocaine; SEM, standard error of the mean; $h$, hours.

Table 2 Trend of the inflammatory infiltrate scores among the different treatments

\begin{tabular}{lll}
\hline Group & Catheter surrounding area $(\mathbf{I ~ c m}):$ median $(\mathbf{I Q R})$ & Contralateral area median $(\mathbf{I Q R})$ \\
\hline A (Saline 0.9\%) & $4(3-4)$ & $0(0-I)$ \\
B (CP I.5\%) & $2(I-2)$ & $0(0-I)$ \\
C (CP 0.5\%) & $3(2-3)$ & $0(0-I)$ \\
\hline
\end{tabular}

Abbreviations: $\mathrm{CP}$, chloroprocaine; IQR, interquartile range.
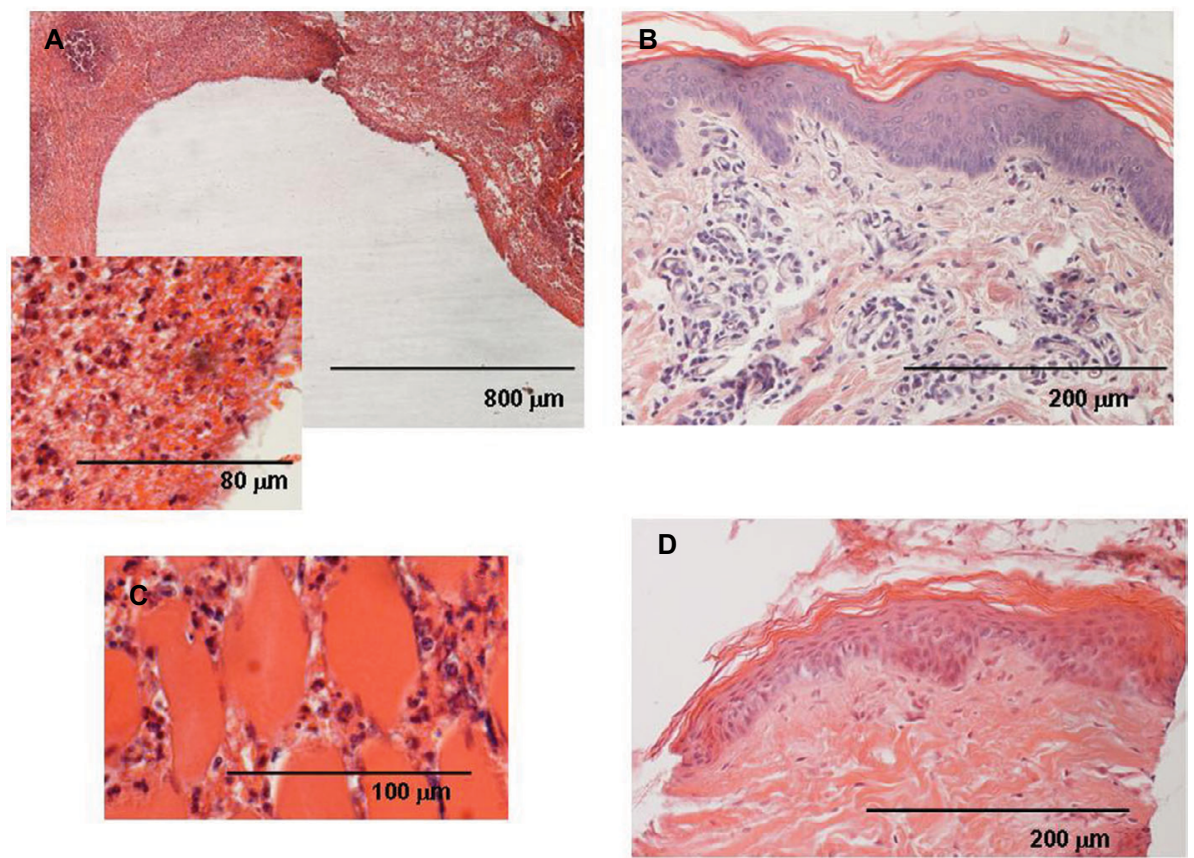

Figure 2 Group A (saline) animal.

Notes: (A) Catheter blank (white in the picture) surrounded by connective tissue. A large amount of inflammatory infiltrate is evident, mostly round cells such as lymphocytes and some PMNs. (Inset) Right next to the blank, many cells appear pale as if suffering or necrotic; collagen fibers are contracted. Many red blood cells are present, probably due to the catheter extraction. (B) Inflammation reaches the cutaneous connective tissue, while (C) a great number of leukocytes surround muscular fibers (D) and contralateral healthy skin appears normal.

Abbreviations: CP, chloroprocaine; PMN, polymorphonuclear granulocyte. 
evident (Figure 3). The reduction in inflammatory infiltrates and signs of tissue regeneration are more evident with higher doses of CP (Figure 4), however no statistically significant difference was observed between groups B and C (Table 2).

\section{Systemic inflammatory analysis}

Plasma cytokine quantification at different time points did not reveal any statistical differences between groups B and $\mathrm{C}$ versus group $\mathrm{A}$, respectively, for the pro-inflammatory
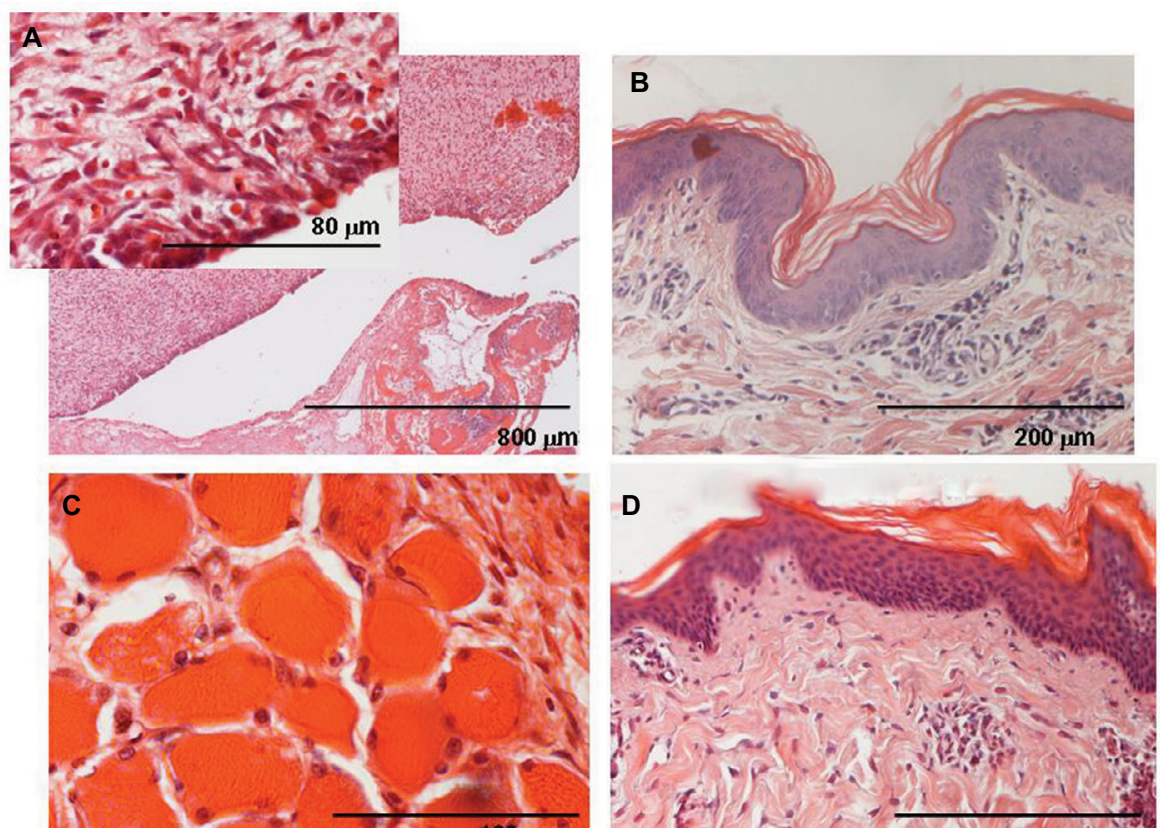

Figure 3 Group B (CP I.5\%) animal.

Notes: (A) Connective wall surrounding the blank shows no structural damage in comparison with controls; inflammatory infiltrate appears less evident, just few leukocytes are present, mostly lymphocytes; (inset) fibroblasts look plump and basophilic; collagen bundles are thin with an irregular arrangement and some tiny blood vessels are evident, suggesting tissue repair. (B) Cutaneous tissues contain a slightly higher amount of infiltrate than contralateral healthy skin (D). (C) Muscular fibers are surrounded by few leukocytes.

Abbreviation: $\mathrm{CP}$, chloroprocaine.
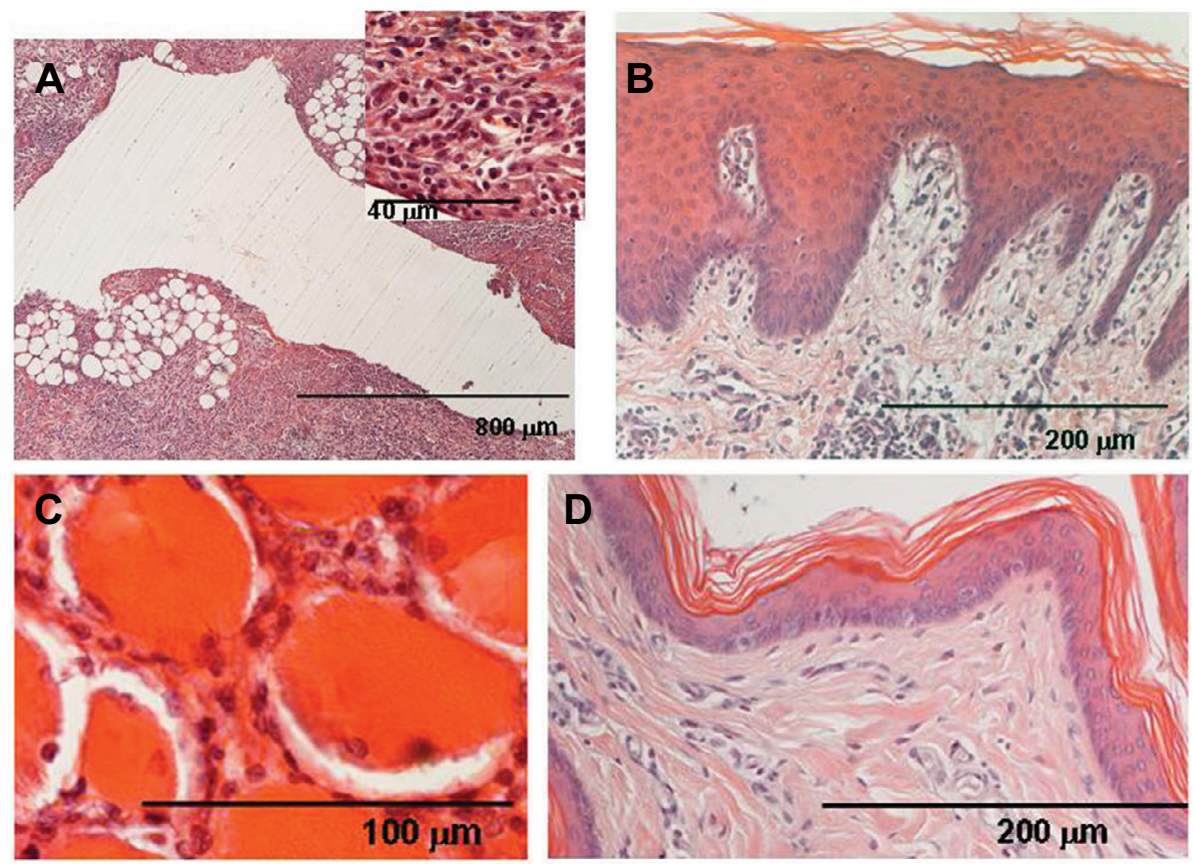

Figure 4 Group C (CP 0.5\%) animal.

Notes: (A) Connective tissue of the blank wall contains active basophilic fibroblasts, thin collagen bundles and a number of round white blood cells (inset); (B) mild inflammatory infiltrate affects dermal papillae and (C) intramuscular connective. (D) Contralateral healthy skin appears normal.

Abbreviation: $\mathrm{CP}$, chloroprocaine. 
cytokines' levels (IL-1 $\beta, p=0.8$ and 0.4 ; IL-6, $p=0.7$ and 0.9 ; IL-8, $p=0.6$ and 0.6 ; IFN- $\gamma, p=0.06$ and 0.2 ) and those of the anti-inflammatory ones (IL-10, $p=0.3$ and 0.3 ) during the entire follow-up period (Figure 5).

\section{In vitro and ex vivo cell proliferation analyses}

The effect of $\mathrm{CP}$ and its metabolite on the in vitro proliferation of pig MNCs stimulated with PHA was evaluated at
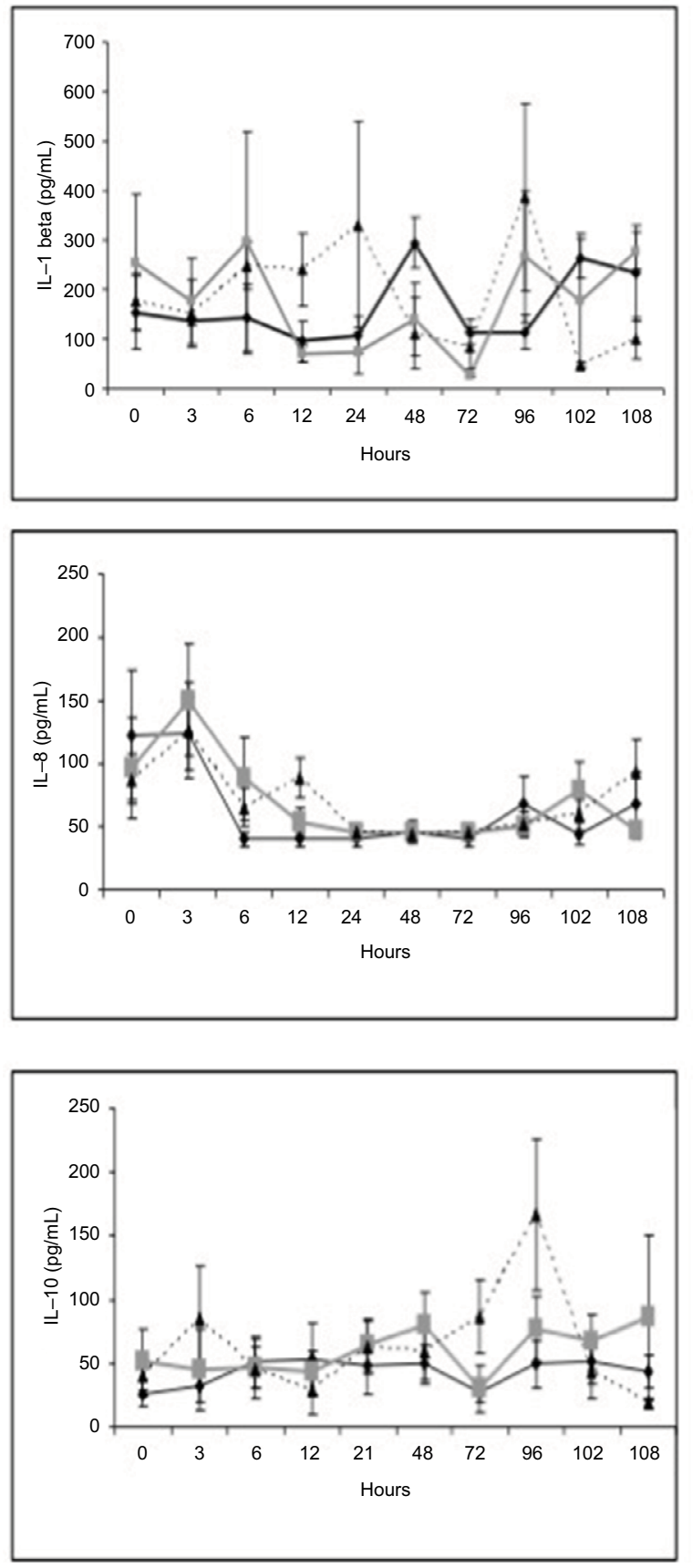

Figure 5 Trend of the blood cytokines levels in the three experimental groups. Notes: Group A, saline; Group B, CP I.5\%; Group C, CP $0.5 \%$.

Abbreviations: $\mathrm{CP}$, chloroprocaine; IL, interleukin; IFN, interferon. different concentrations in 4 animals using MNCs obtained before any kind of surgical procedure and treatment. The results are shown in Figure 6.

PHA-stimulated proliferation of MNCs was significantly reduced by the addition of 1.25 and $0.6 \mathrm{mg} / \mathrm{mL}$ of $\mathrm{CP}$, with a direct correlation between the concentrations and the degree of inhibition. A decrease in proliferation was also observed for CABA at $1.25 \mathrm{mg} / \mathrm{mL}$, but it was not statistically significant $(p=0.1)$.
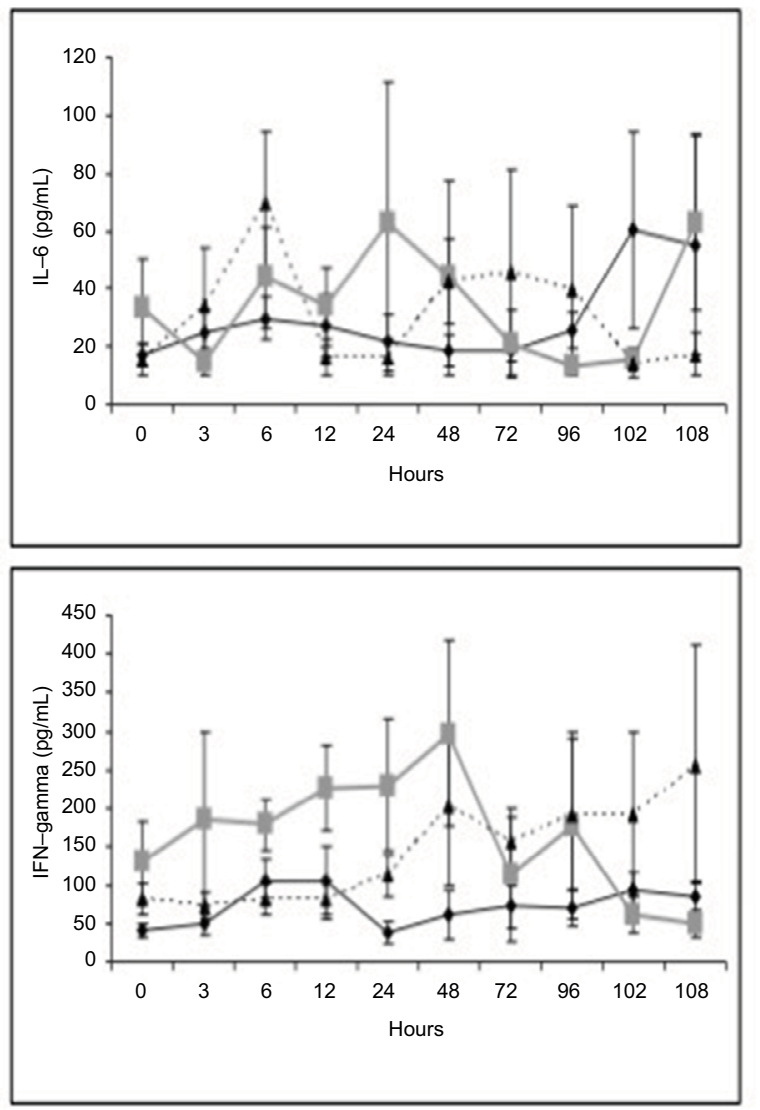
A

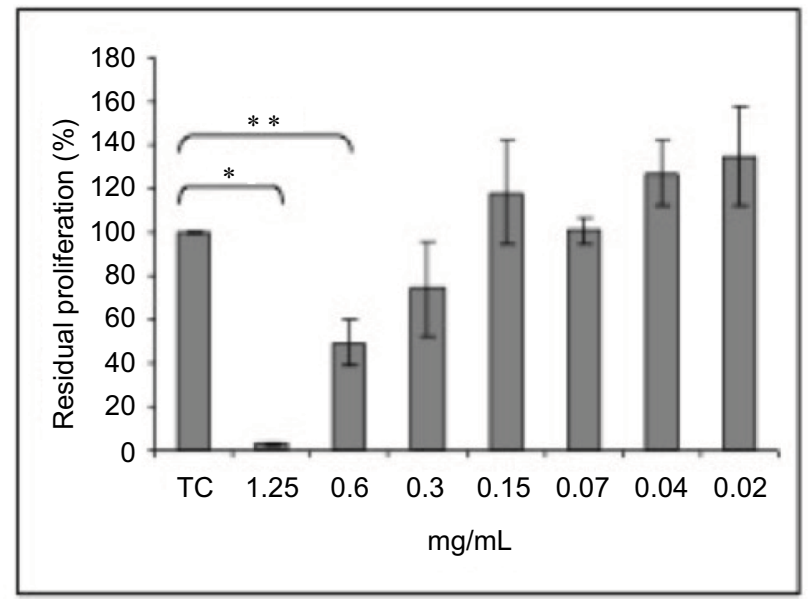

B

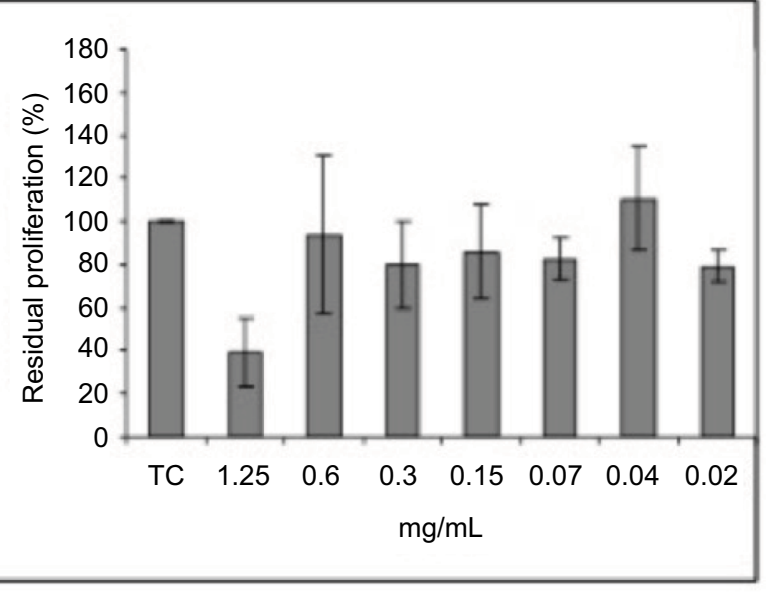

C

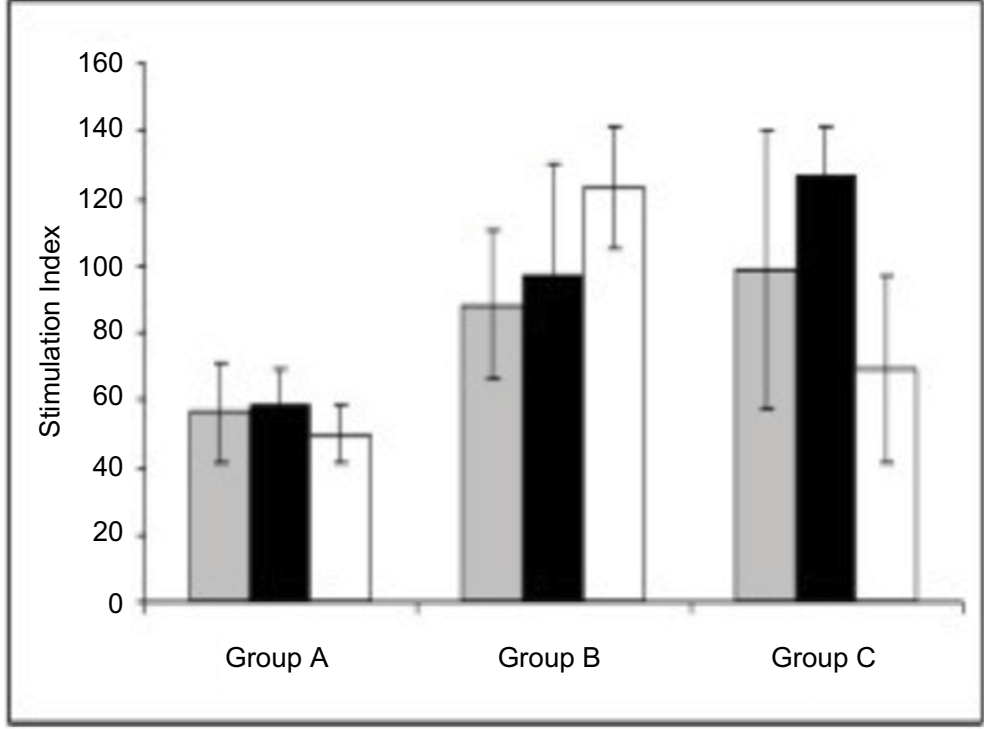

Figure 6 Treatment group in vitro proliferation.

Notes: In vitro cell proliferation suppression by CP $(\mathbf{A})$ and its metabolite (B) at different concentrations, on PHA-activated pig MNCs. Results are expressed as residual proliferation. CP induces significant reduction at concentration of $1.25 \mathrm{mg} / \mathrm{mL}\left({ }^{*} p=0.012\right.$ ) and of $0.6 \mathrm{mg} / \mathrm{mL}(* * p=0.052)$. (C) In vitro PHA-induced proliferation of MNCs obtained from group A, B, C animals at different time points (gray bars $=$ T0, black bars $=96$ hours and white bars $=108$ hours). Results are expressed as stimulation index. No significant differences are observed between treated and control groups. Group A, saline; Group B, CP I.5\%; Group C, CP 0.5\%.

Abbreviations: $\mathrm{CP}$, chloroprocaine; MNCs, mononuclear cells; PHA, phytohemagglutinin.

Evaluation of cell viability, by Trypan blue exclusion test, showed that the addition of $\mathrm{CP}$ and CABA to in vitro resting and activated pig MNCs did not cause cell death, even at the highest concentration (percentage of viable cells $72 \%$ and $79 \%$, respectively), suggesting the lack of cytotoxic activity of $\mathrm{CP}$ and its metabolite. Moreover, $\mathrm{CP}$ and CABA did not induce in vitro cell proliferation at any concentrations of resting MNCs, indicating no antigenic activity.

In order to assess the effect of CP and CABA we evaluated ex vivo the response to PHA of MNCs isolated from blood collected at different time points (basal, 96 and 108 hours). Results did not show any significant differences between group A versus group B $(p=0.22)$ and versus group C $(p=0.34)$ at each time point.

\section{Discussion}

So far, long-acting LAs have mainly been used for CWI, despite their suboptimal pharmacokinetic profile and the lack of studies investigating their systemic exposure.

New formulations of CP, an ultrashort acting LA with plasmatic pseudocholinesterase metabolism, theoretically display an ideal pharmacokinetic profile for CWI. In a translational perspective, and responding to the need of testing this molecule in an experimental setting, we investigated the use of CP in a large animal model (pig).

The current investigation confirmed the hypothesis that CWI in our large animal model would result in relatively low amounts of $\mathrm{CP}$ being absorbed, and in light of the extremely 
short half-life of CP in plasma may definitely limit the possibility of systemic effects: indeed during the 96 hours of $5 \mathrm{~mL} / \mathrm{h}$ continuous administration of both $0.5 \%$ and $1.5 \%$ $\mathrm{CP}$, the overall amount of CP penetrating the systemic circulation (that is plasma concentrations of $\mathrm{CP}+$ its plasmatic metabolite CABA) reached an average $C_{\max }$ at least 10 -fold lower than that observed in obstetric patients receiving epidural CP during cesarian section. ${ }^{24} \mathrm{~A}$ dose-related increase in concentrations was observed after the beginning of the CWI and up to 6 hours, suggesting an initial blood vesseldilating response to $\mathrm{CP},{ }^{25}$ resulting in a peak of absorption that appeared to progressively decrease over time. We registered no side effects or allergic reaction in any of the enrolled animals. Our study confirms $\mathrm{CP}$ as an ideal local anesthetic for CWI, due to its fast catabolism, safety and on/off profile.

The lack of a cholinesterase inhibitor at collection prevents the actual assessment of systemic exposure to intact $\mathrm{CP}$ since $\mathrm{CP}$ degradation occurs very rapidly in plasma, but reporting the total amount of $\mathrm{CP}$ entering the bloodstream (that is $\mathrm{CP}+\mathrm{CABA}$ ) our study reliably describes the overall absorption of CP upon CWI, showing concentrations well below those observed upon epidural administration during either cesarean section or vaginal delivery, ${ }^{24}$ peaking between 3 and 6 hours and decreasing to undetectable levels after infusion was suspended. Therefore, also taking into account potential differences in esterase activities between the experimental animal used and humans, the systemic exposure to CP appeared very limited, as confirmed by the lack of effects on inflammatory parameters or blood cell activity. Indeed no correlation was detected between treatment and systemic inflammation, as assessed by determination of plasma concentrations of pro-inflammatory cytokines, suggesting that only local inflammation was modulated by the presence of CP. Additionally, local infusion of CP did not affect the ex vivo response of immune cells obtained from treated animals, even if PHA-induced proliferation of MNCs was suppressed by in vitro addition of $\mathrm{CP}$, further supporting the lack of significant circulating concentrations of intact $\mathrm{CP}$ as suggested by the relatively low plasmatic concentrations of CABA.

Our data also suggest that the continuous infusion with CP displays local anti-inflammatory effects. These effects were dose-dependent, as we observed a more pronounced reduction of inflammatory response in the group treated with higher concentrations of local anesthetic. Since we did not monitor pain, we cannot determine if the dose-dependent anti-inflammatory effect is associated with either better pain relief, or with a more pronounced reduction of neurogenic inflammation or with specific anti-inflammatory effect of CP. Investigating the pain response would have provided additional support to our studies, but measuring pain in a large animal model can be very challenging, and a well-accepted model is still lacking. Nevertheless, apart from the assessment of the analgesic effect, our study was able to confirm in a large animal model results previously obtained only in small animals, ${ }^{26,27}$ ie, the ability of nociceptive block to reduce local inflammation. In consideration of the promising results relative to the limited absorption and the anti-inflammatory effect of CP obtained with this study, future trials are warranted to address this topic.

Despite the low sample size (typical of studies in big animal models ${ }^{19}$ ), we confirmed the limited absorption and a local anti-inflammatory activity of CWI with CP. Once these results are confirmed in humans, they may show $\mathrm{CP}$ to be a good choice for CWI allowing immediate analgesic benefit and fast elimination; patients with liver or kidney diseases may take advantage of rapid plasmatic clearance of $\mathrm{CP}$, preventing accumulation and toxicity. Clinical studies are needed to define the lowest effective dose of CP and investigate its anti-inflammatory effect in a human model.

\section{Conclusion}

This pilot study on CP continuous infusion in a large animal model may display future translational value. Once confirmed in humans, our data may show CP to be a first-line choice for CWI, with limited systemic exposure, high compliance to the needs of each patient and potential applications to a broad spectrum of clinical situations. The dose-dependent anti-inflammatory effect may have clinical relevance in pain medicine, showing CP to be an interesting target for future clinical applications and research purposes.

\section{Acknowledgment}

The authors would like to thank the following colleagues as strong contributors to this study: Tommaso Dominioni and Andrea Peloso - Department of Surgical, Clinical, Paediatric and Diagnostic Science, University of Pavia, and General Surgery 1, IRCCS Fondazione Policlinico San Matteo, Pavia, Italy; Andrea Fanelli - Department of Anaesthesiology and Intensive Care, Azienda OspedalieroUniversitaria Policlinico S. Orsola-Malpighi, Bologna, Italy; Daniela Ghisi - Department of Anaesthesia and Postoperative Intensive Care, Istituto Ortopedico Rizzoli, Bologna, Italy; Giovanna Riccipetitoni, Department of Pediatric Surgery, "V. Buzzi” Children's Hospital, Milan, Italy; Patrizia Risé - Department of Pharmacological and 
Biomolecular Sciences, University of Milan. The study has been funded by a grant by the Italian Health Ministry ("New nanotechnology and biomedical approaches to improve postoperative pain treatment reducing risks related to opioids" - GR-2010-2318370; Principal Investigator Massimo Allegri, MD) and a grant by Foundation "Banca del Monte di Lombardia" ("La Nanotecnologia applicata al Trattamento del dolore"; Principal Investigator Lorenzo Cobianchi, MD). The authors would like to thank Sintetica ${ }^{\circledR}$ for providing Chloroprocaine for this study.

\section{Disclosure}

The authors report no conflicts of interest in this work.

\section{References}

1. Coluzzi F, Savoia G, Paoletti F, Costantini A, Mattia C. Postoperative pain survey in Italy (POPSI): a snapshot of current national practices. Minerva Anestesiol. 2009;75(11):622-631.

2. Benhamou D, Berti M, Brodner G, et al. Postoperative Analgesic THerapy Observational Survey (PATHOS): a practice pattern study in 7 central/southern European countries. Pain. 2008;136(1-2):134-141.

3. Romundstad L, Stubhaug A. Glucocorticoids for acute and persistent postoperative neuropathic pain: what is the evidence? Anesthesiology. 2007;107(3):371-373.

4. Bugada D, Lavand'homme P, Ambrosoli AL, et al. Effect of preoperative inflammatory status and comorbidities on pain resolution and persistent postsurgical pain after inguinal hernia repair. Mediators Inflamm. 2016;2016:5830347.

5. Burian M, Geisslinger G. COX-dependent mechanisms involved in the antinociceptive action of NSAIDs at central and peripheral sites. Pharmacol Ther. 2005;107(2):139-154.

6. Hains LE, Loram LC, Weiseler JL, et al. Pain intensity and duration can be enhanced by prior challenge: initial evidence suggestive of a role of microglial priming. J Pain. 2010;11(10):1004-1014.

7. Allegri M, Grossi P. Management of postoperative pain: how accurate and successful is our acute pain management? Minerva Anestesiol. 2012;78(1):1-3.

8. Lavand'homme P. From preemptive to preventive analgesia: time to reconsider the role of perioperative peripheral nerve blocks? Reg Anesth Pain Med. 2011;36(1):4-6.

9. Campos CR, Ocheltree SM, Hom S, Egleton RD, Davis TP. Nociceptive inhibition prevents inflammatory pain induced changes in the bloodbrain barrier. Brain Res. 2008;1221:6-13.

10. Fu KY, Light AR, Maixner W. Relationship between nociceptor activity, peripheral edema, spinal microglial activation and long-term hyperalgesia induced by formalin. Neuroscience. 2000;101(4):1127-1135.
11. Bugada D, De Gregori M, Compagnone C, et al. Continuous wound infusion of local anesthetic and steroid after major abdominal surgery: study protocol for a randomized controlled trial. Trials. 2015;16:357.

12. Lavand'homme P. Improving postoperative pain management: continuous wound infusion and postoperative pain. Eur J Pain Suppl. 2011;5(2): 315-512.

13. Ventham NT, Hughes M, O’Neill S, Johns N, Brady RR, Wigmore SJ. Systematic review and meta-analysis of continuous local anaesthetic wound infiltration versus epidural analgesia for postoperative pain following abdominal surgery. Br J Surg. 2013;100(10):1280-1289.

14. Cassuto J, Sinclair R, Bonderovic M. Anti-inflammatory properties of local anesthetics and their present and potential clinical implications. Acta Anaesthesiol Scand. 2006;50(3):265-282.

15. Perotti L, Cusato M, Ingelmo P, et al. A comparison of differences between the systemic pharmacokinetics of levobupivacaine and ropivacaine during continuous epidural infusion: a prospective, randomized, multicenter, double-blind controlled trial. Anesth Analg. 2015;121(2): 348-356.

16. Warren DT, Kopacz DJ. Spinal 2-chloroprocaine: the effect of added dextrose. Anesth Analg. 2004;98(1):95-101. table of contents.

17. Moore DC, Spierdijk J, vanKleef JD, Coleman RL, Love GF. Chloroprocaine neurotoxicity: four additional cases. Anesth Analg. 1982;61(2): 155-159.

18. Taniguchi M, Bollen AW, Drasner K. Sodium bisulfite: scapegoat for chloroprocaine neurotoxicity? Anesthesiology. 2004;100(1):85-91.

19. Gigliuto C, De Gregori M, Malafoglia V, et al. Pain assessment in animal models: do we need further studies? J Pain Res. 2014;7:227-236.

20. Cobianchi L, Zonta S, Vigano J, et al. Experimental small bowel transplantation from non-heart-beating donors: a large-animal study. Transplant Proc. 2009;41(1):55-56.

21. Kaiser GM, Heuer MM, Fruhauf NR, Kuhne CA, Broelsch CE. General handling and anesthesia for experimental surgery in pigs. J Surg Res. 2006;130(1):73-79.

22. Dhananjeyan MR, Bykowski C, Trendel JA, Sarver JG, Ando H, Erhardt PW. Simultaneous determination of procaine and para-aminobenzoic acid by LC-MS/MS method. J Chromatogr B Analyt Technol Biomed Life Sci. 2007;847(2):224-230.

23. Zientek KD, Anderson DF, Wegner K, Cole C. The quantitation of procaine in equine plasma by liquid chromatography-linear ion trap mass spectrometry. J Anal Toxicol. 2007;31(2):87-92.

24. Kuhnert BR, Kuhnert PM, Prochaska AL, Gross TL. Plasma levels of 2-chloroprocaine in obstetric patients and their neonates after epidural anesthesia. Anesthesiology. 1980;53(1):21-25.

25. Covino BG. Pharmacology of local anaesthetic agents. Br J Anaesth. 1986;58(7):701-716.

26. Beloeil H, Gentili M, Benhamou D, Mazoit JX. The effect of a peripheral block on inflammation-induced prostaglandin E2 and cyclooxygenase expression in rats. Anesth Analg. 2009;109(3):943-950.

27. Combettes E, Benhamou D, Mazoit JX, Beloeil H. Comparison of a bupivacaine peripheral nerve block and systemic ketoprofen on peripheral inflammation and hyperalgesia in rats. Eur J Anaesthesiol. 2010;27(7): $642-647$.
Journal of Pain Research

\section{Publish your work in this journal}

The Journal of Pain Research is an international, peer reviewed, open access, online journal that welcomes laboratory and clinical findings in the fields of pain research and the prevention and management of pain. Original research, reviews, symposium reports, hypothesis formation and commentaries are all considered for publication.

\section{Dovepress}

The manuscript management system is completely online and includes a very quick and fair peer-review system, which is all easy to use. Visit http://www.dovepress.com/testimonials.php to read real quotes from published authors. 